\title{
HexGraph: Applying Graph Drawing Algorithms to the Game of Hex
}

\author{
Colin Murray, Carsten Friedrich, and Peter Eades \\ School of Information Technologies, The University of Sydney, Australia \\ $\{$ cmurray, carsten, peter\}@it.usyd.edu.au
}

Hex [1] is a two player board game which is traditionally played on a rhombic hexagonal pattern (See Figure 1). Players are assigned a colour and make moves by putting a token of their colour onto an empty field on the board. The first player to connect the two borders of the board in his colour by a path of his tokens on the board wins the game. Alternatively, Hex is played on an undirected, tricoloured (Red, Blue, Unclaimed) graph $G$ [2]. The fields are represented by nodes and adjacent fields on the board are connected by an edge. The four borders of the board are represented by one node of equivalent colour each (See Figure [1).
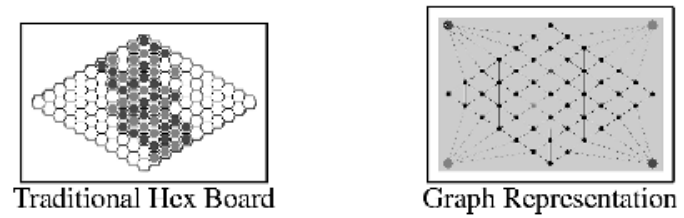

Fig. 1. Traditional (Source [2]) \& Graph representations.

Making a move triggers the following transformation $f$ on the graph:

1. Edges to nodes held by the opponent are obsolete and are removed from the graph.

2. Edges which connect nodes of the same colour can be contracted.

3. Isolated nodes, that is, nodes which do not have any two non-intersecting paths to the two border nodes are obsolete (they cannot be part of a shortest winning path) and can thus be removed from the graph.

The graph continually simplifies during the course of the game. The challenge is to make the drawing simpler as well, and thus making it easier to play a winning strategy. Note that the transformation $f$ preserves planarity. However, simple minded contraction of edges introduces edge-crossings. The HexGraph paradigm uses planar and embedding-preserving graph drawing algorithms, in combination with the graph transformation $f$ above. We have experimented with a number of approaches. The best results are achieved by combining the Tutte [3.4 and the force directed PrEd [5] algorithms. The edge-crossings are removed by the Tutte algorithm and then the force directed PrEd algorithm 
improves symmetry and uniformity of edge lengths without introducing any edge crossings. These graph drawing features help to ensure that the player understands the state of the game and should improve the player's ability to play a winning strategy.

We also use graph animation [6] to show the transition from the initial graph drawing to the final graph drawing to help the user maintain the mental map [7] of the game.

In our experience, the HexGraph paradigm provides great potential to increase a player's ability to find good moves and strategies in a game of hex. Our implementation is available from http://www.it.usyd.edu.au/ ${ }^{\sim}$ carsten/hex

(a)
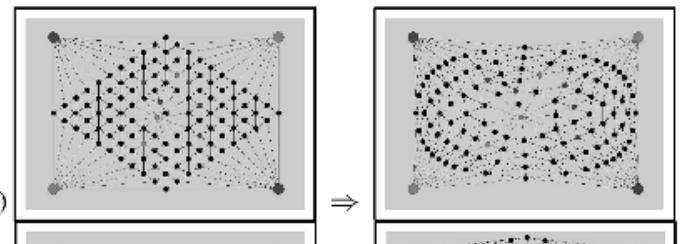

(b)
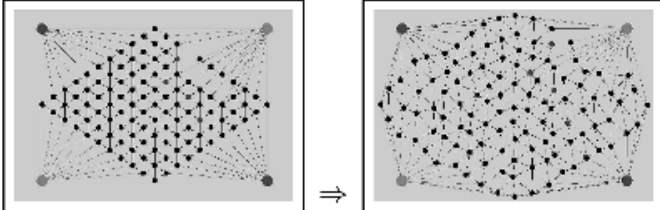

(c)
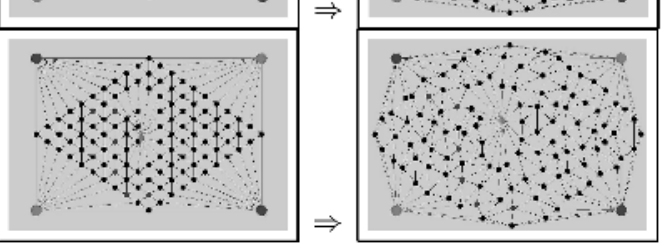

Fig. 2. (a) The Tutte Algorithm removes edges crossings from the graph. (b) Force directed PrEd makes edge crossings less confusing. (c) The edge-crossings are removed by the Tutte algorithm and then the force directed PrEd algorithm improves symmetry and uniformity of edge lengths

\section{References}

1. C. Browne. Hex Strategy: Making the Right Connections. A. K. Peters, Natick, Massachusetts, 2000.

2. Jack van Rijswijck. Search and evaluation in Hex. Tech report, University of Alberta.

3. W. T. Tutte. Convex representations of graphs. Proc. Lond. Math. Soc., 10:304-320, 1960.

4. W. T. Tutte. How to draw a graph. Proc. London Math Soc., 13:743-768, 1963.

5. F. Bertault. A force-directed algorithm that preserves edge-crossing properties. Information Processing Letters, 74(1-2):7-13, 2000.

6. Carsten Friedrich. Animation in Relational Information Visualization. Phd thesis, University of Sydney, 2002.

7. P. Gould and R. White. Mental Maps. Allen and Unwin, Winchester, 1986. 